\title{
Selection and Characteristics of Fermented Salted Seafood (jeotgal)-Originated Strains with Excellent S-adenosyl-L-methionine (SAM) Production and Probiotics Efficacy
}

\author{
Min-Jeong Kim, Sunhyun Park, Ran-Sook Lee, Sang-Dong Lim, Hyo Jin Kim, and Myung-Ki Lee* \\ Korea Food Research Institute, Seongnam 463-746, Korea
}

\begin{abstract}
This study is executed to develop probiotics which produce S-adenosyl-L-methionine (SAM), a methyl group donor of the 5-methyltetrahydrofolate methylation reaction within the animal cell. SAM is an essential substance for the synthesis, activation, and metabolism of hormones, neurotransmitters, nucleic acids, phospholipids, and cell membranes of animals. The SAM is also known as a nutritional supplement to improve brain functions of the human. In this study, the SAM-producing strains are identified in 18 types of salted fish, and then, the strains with excellent SAM productions are being identified, with 1 strain in the Enterococcus genus and 9 strains in the Bacillus genus. Strains with a large amount of SAM production include the lactic acid bacteria such as En. faecium and En. durans, En. sanguinicola, as well as various strains in the Bacillus genus. The SAM-overproducing strains show antibacterial activities with certain harmful microbes in addition to the weak acid resistances and strong bile resistances, indicating characteristics of probiotics. It is possible that the jeotgal-originated beneficial strains with overproducing SAM can be commercially utilized in order to manufacture SAM enriched foods.
\end{abstract}

Key words: S-adenosyl-L-methionine, fermented salted seafood probiotics, jeotgal

\section{Introduction}

S-adenosyl-L-methionine (SAM), first discovered by Cantoni in 1952, is a substance primarily present in tissues and body fluids of an organism, and it plays an important role in the 5-methyltetrahydrofloat (5-MTHF) methylation reaction as a methyl group donor (Shelly, 2000). SAM is an important bio-regulator made from essential amino acids, L-methionine, and ATP by methionine adenosyltransferase (Wang et al., 2001). It is mostly synthesized in the liver at a rate of about $8 \mathrm{~g}$ a day and is involved in many biochemical metabolism processes (Horikawa et al., 1990).

Through many studies, it has been confirmed that SAM content varies depending on the content of L-methionine in foods (Kim et al., 2008). Its functionality has been recognized in western societies including Europe and the USA, and SAM has been reported as an essential substance in processes including in polyamine synthesis in

*Corresponding author: Myung-Ki Lee, Korea Food Research Institute, Seongnam 463-746, Korea. Tel: 82-31-780-9045, Fax: 82-31-709-9876, E-mail: lmk123@kfri.re.kr cells and the synthesis, activation, and metabolism of hormones, neurotransmitters, nucleic acids, phospholipids, and cell membranes (Santi et al., 1983). Also, it is required in the production of important brain compounds such as neurotransmitters and phospholipids, including phosphatidylcholine and phosphatidylserine, and is known to be effective in improving brain functions (Mato et al., 1990).

The metabolic reactions of SAM in the body include transmethylation, transsulfuration, and polyamine (Mato et al., 1999). In the methylation reaction, the methyl group of SAM is donated to various acceptor substrates such as DNA, phospholipids, and proteins; in the transsulfuration reaction, SAM is converted to taurine, a major antioxidant in cells, and cysteine, a precursor of glutathione, through several enzymatic reactions (Porter et al., 1986). Finally, SAM is used in the synthesis of polyamines that are essential to the growth of normal cells (Cooney, 1993). It also donates the methyl group to the propolyamine group in the synthesis of spermine and spermidine (Lee et al., 2006). It has been reported that SAM has pharmacological effects in the restoration of hepatic functions in alcoholic liver disease, in reducing muscle fatigue and 
rigidity in patients with fibromyalgia syndrome, and in improvement in patients with depression with about a 66\% clinical improvement (Koning, 1987; Lieber, 1999).

Up to now, studies on SAM reported its effects in the treatment of depression, and in arthritis and hepatic cirrhosis, and it has been recognized as a health-functional food with a recommended daily intake of $400 \mathrm{mg}$ (Lee et al., 2008). A food containing SAM includes Jeotgal (fermented salted seafood), one of our traditional fermented foods. Thus, this study was performed to isolate jeotgaloriginated strains that produce physiologically functional material, SAM, select and identify strains that produce large amounts of SAM in foods, and find strains producing SAM with probiotics through antibacterial activity to function against harmful microbes such as food poisoning bacteria.

\section{Materials and Methods}

\section{Sample preparation}

Naturally existing strains in 18 kinds of jeotgal were isolated and their colony was analyzed to select strains that improve SAM production. Samples used in the study included 18 types of fermented salted seafood (toha shrimp, whitesaddled reeffish, baby octopus, herring roe, scallop, clam, pen-shell, hairtail, gizzard shad, hairtail guts, sand lance sauce, large-eyed herring, branchia, shrimp, anchovy, squid, small octopus, pollack roe) purchased in Ganggyeong, Chungcheongnam-do (April 18, 2012).

\section{Characteristics of selected strains}

Samples were diluted 10 times $(0.85 \% \mathrm{NaCl} 225 \mathrm{~mL}+$ sample $25 \mathrm{~g}$ ) under a sterilized environment and then homogenized using a stomacher (speed level 5, $1 \mathrm{~min}$ ). Then, the homogenate was smeared on each selective medium and incubated to separate total microbes, lactic acid bacteria, anaerobic bacteria, yeast and mold. Microbial separation and collection were performed depending on the colony types of microbes produced. Microbes grown in different selective media were identified. The separation of microbes used tryptic soy agar (TSA) for analysis of the number of total microbes. In addition, for the analysis of total lactic acid bacteria, MRS agar with adjusted $\mathrm{pH}$ 5.5 was used. For a detailed analysis of the lactic acid bacteria, m-LBS (Lactobacillus species), KF-Streptococcus (Enterococcus, Pediococcus species) and PES (Leuconostoc species) were used. Also, potato dextrose agar (PDA) with an adjusted $\mathrm{pH}$ using tartaric acid was used for the analysis of the yeast and mold (Table 1).
Table 1. Selective medium type and isolated colony

\begin{tabular}{|c|c|}
\hline Medium type & Target bacteria \\
\hline $\mathrm{TSA}^{1)}($ Merck$)$ & Total microbes \\
\hline $\mathrm{MRS}^{2)}($ adjust pH 5.5) & Total LAB \\
\hline KF-Streptococcus ${ }^{3)}$ & Enterococcus/Pediococcus \\
\hline$m-\mathrm{LBS}^{4)}$ & Lactobacillus \\
\hline $\mathrm{PES}^{5)}$ & Leuconostoc \\
\hline $\begin{array}{l}\text { PDA }^{6)} \text { (Difco, adjust } \mathrm{pH} \text { using } \\
\text { tartaric acid solution) }\end{array}$ & Yeast and mold \\
\hline \multicolumn{2}{|l|}{ 1)TSA: Tryptic soy agar } \\
\hline \multicolumn{2}{|l|}{${ }^{2)} \mathrm{MRS}$ : Lactobacilli MRS agar } \\
\hline \multicolumn{2}{|c|}{${ }^{3)} \mathrm{KF}$ : KF- streptococcus } \\
\hline \multicolumn{2}{|c|}{${ }^{4)}$ m-LBS: modified Lactobacillus selection agar } \\
\hline \multicolumn{2}{|c|}{${ }^{5)} \mathrm{PES}$ : phenylethyl alcohol with $2 \%$ sucrose agar } \\
\hline${ }^{6)}$ PDA: Potato dextrose agar & \\
\hline
\end{tabular}

\section{Culture of strains isolated from jeotgal}

To examine SAM production ability, strains were inoculated onto a nutrient medium TSB, incubated at $35^{\circ} \mathrm{C}$ for $24-48 \mathrm{~h}$ to maintain $10^{8}-10^{9} \mathrm{CFU} / \mathrm{mL}$, and centrifuged $\left(4,000 \mathrm{rpm}, 4^{\circ} \mathrm{C}, 10 \mathrm{~min}\right)$ to eliminate microbial cells and collect the supernatant for analysis.

\section{SAM production}

The production of SAM was analyzed using high-performance liquid chromatography (HPLC) under the conditions listed in the table (Guattari, 1991; Katie et al., 2006). Prepared samples were filtered using a $0.45 \mu \mathrm{m}$ syringe filter and stored at $-20^{\circ} \mathrm{C}$ for analysis. The standard for SAM was purchased from Sigma-Aldrich, and the ammonium acetate and methanol used in the analysis and all other reagents were purchased for HPLC use.

\section{Analysis of sugar fermentation by isolated strains}

Among the isolated strains, the Enterococcus genus strains used an API 20 Strep system kit (bioMereux, France) and the Bacillus genus strains used an API 50 CHB system kit (bioMereux, France) for the analysis of the sugar fermentation of the strains. According to the API kit manual, diluted strain samples were transferred to each selective medium and incubated at $37^{\circ} \mathrm{C}$ for $24-48 \mathrm{~h}$ to identify the presence/absence of various sugar fermentation.

\section{Acid resistance and bile resistance of strains}

For the resistance to $\mathrm{pH}$, strains were activated in TSB $\left(10^{8}-10^{9} \mathrm{CFU} / \mathrm{mL}\right)$, washed with $\mathrm{PBS}$ or $0.85 \% \mathrm{NaCl}$, and then centrifuged for sample use (centrifuge use). After TSA medium preparation with $\mathrm{pH}$ levels of 2.0, 2.5, 3.0, 3.5, 4.0, 4.5, 5.0, and 5.5 (control pH 7.0 - medium $\mathrm{pH}$ ), samples were spotted on TSA and changes of the colony 
Table 2. HPLC conditions for SAM analysis

\begin{tabular}{cc}
\hline \hline Item & Condition \\
\hline HPLC & Jasco Co., Japan \\
& Pump : PU-980 \\
Detector : UV-975 \\
Column oven : CO-965 \\
Auto sampler : AS-2057 \\
Column & Shiseido C18 $(4.6 \times 205 \mathrm{~mm}, 5 \mu \mathrm{m})$ \\
& A: $100 \%$ Methanol \\
Mobile phase & B: $0.25 \mathrm{M}$ Ammonium acetate $(\mathrm{pH} 5.5)$ \\
& with acetic acid \\
Temperature & $40^{\circ} \mathrm{C}$ \\
Velocity & $1.5 \mathrm{~mL} / \mathrm{min}$ \\
Detector & $\mathrm{UV} \mathrm{detector}$ \\
Wavelength & $254 \mathrm{~nm}$ \\
\hline
\end{tabular}

after 18-24 h were measured. Similarly, for the bile resistance of strains, the resistance of each strain was measured in the medium with or without oxgall. After strain activation on TSB $\left(10^{8}-10^{9} \mathrm{CFU} / \mathrm{mL}\right)$, samples were washed with PBS or $0.85 \% \mathrm{NaCl}$ (centrifuge use), $1 \%$ of the washed strain was inoculated onto the medium with or without $0.3 \%$ oxgall and spotted on TSA, and the changes of the colony were measured after 18-24 h.

\section{Antibacterial activity of strains}

The activity of strains that inhibited five of the indicators including Escherichia coli O157:H7, Enterococcus faecalis, Salmonella choleraesuis, Staphylococcus aureus, and Listeria monocytogenes were measured. Similarly in the soft agar method, strains were activated and spotted on TSA and incubated for $12 \mathrm{~h}$ in an incubator. Then, $10 \mathrm{~mL}$ of soft agar with $0.75 \%$ agar concentration was prepared and indicators were added to the agar at $50^{\circ} \mathrm{C}$ to reach $10^{5}$ $\mathrm{CFU} / \mathrm{mL}$ before it was hardened. It was evenly spotted onto TSA with grown strains, and the clear zone of circle around the colony was observed every $4 \mathrm{~h}$.

\section{Identification of isolated strains by $16 \mathrm{~S}$ rRNA se- quence analysis}

Strains with a higher production of SAM were identified by Macrogen Inc. (Korea). Genomic DNA was extracted from each strain and was used for the PCR amplification of $16 \mathrm{~S}$ rRNA gene.

\section{Results and Discussion}

\section{Isolation of jeotgal-originated colonies}

The microbes isolated using various selective medium were sorted by the morphology of colony. The colony was analyzed using 18 kinds of jeotgal as shown in the Table below. Microbes were grouped by shape, color, and characteristics, and a total of different 169 colony types were classified (Table 3).

\section{SAM production}

The amount of SAM production was measured in 169 strains isolated from 18 kinds of jeotgal as shown in Table 3. Among 169 strains, 10 strains with excellent SAM production were selected from strains that had originated from each jeotgal. And then, the strains were identified by $16 \mathrm{~S}$ rRNA sequencing at Macrogen Co. and the population size were detected (Table 4). As a result, 9 strains of the Bacillus genus and 1 strain of the Enterococcus genus were identified.

The Bacillus genus, which is known as the major fermentation strain in jeotgal, used a method for all the microbes using a TSA medium; although yeast and molds, in addition to lactic acid bacteria, were separated using a selective medium in order to discover and utilize strains with various ecosystems in jeotgal, most of the dominant microbes were in the Bacillus genus and the Enterococcus genus (Hur, 1996; Lee, 1969; Sands and Crisan, 1974). The strain with the maximum production of SAM was a J6-9 strain isolated from salted clam, which produced 1.41

Table 3. Jeotgal-originated strains isolated by colony type

\begin{tabular}{|c|c|c|c|c|c|}
\hline No. & Source name & Number of isolated strains* & No. & Source name & Number of isolated strains \\
\hline 1 & salted toha shrimp & 14 & 10 & salted hairtail guts & 9 \\
\hline 2 & salted white saddled reeffish & 4 & 11 & salted sand lance sauce & 2 \\
\hline 3 & salted baby octopus & 9 & 12 & salted large-eyed herring & 13 \\
\hline 4 & salted herring roe & 14 & 13 & salted branchia & 9 \\
\hline 5 & salted scallop & 9 & 14 & salted shrimp & 4 \\
\hline 6 & salted clam & 9 & 15 & salted anchovy & 3 \\
\hline 7 & salted pen-shell & 14 & 16 & salted squid & 15 \\
\hline 8 & salted hairtail & 5 & 17 & salted small octopus & 10 \\
\hline 9 & salted gizzard shad & 19 & 18 & salted pollack roe & 7 \\
\hline
\end{tabular}

*The strains isolated using various selective medium were sorted by the morphology of colony. 
Table 4. SAM production and identification of strains isolated from various jeotgal

\begin{tabular}{cccccc}
\hline \hline Name & Isolation medium & SAM Production (mM) & Population size (CFU/mL) & Identification result & Identity (\%) \\
\hline J6-9 & MRS & 1.41 & $5.7 \times 10^{5}$ & Enterococcus durans & 99 \\
J7-1 & TSA & 1.37 & $3.1 \times 10^{5}$ & Bacillus sp. & 99 \\
J7-5 & TSA & 1.33 & $9.1 \times 10^{4}$ & Bacillus subtilis & 99 \\
J13-4 & TSA & 1.29 & $1.3 \times 10^{4}$ & Bacillus pumilus & 99 \\
J8-4 & TSA & 1.27 & $9.7 \times 10^{4}$ & Bacillus amyloliquefaciens & 99 \\
J7-10 & mLBS & 1.23 & $2.9 \times 10^{5}$ & Bacillus amyloliquefaciens & 99 \\
J5-9 & mLBS & 1.16 & $7.4 \times 10^{5}$ & Bacillus sp. & 99 \\
J8-3 & TSA & 1.07 & $2.1 \times 10^{5}$ & Bacillus methylotrophicus & 99 \\
J4-3 & TSA & 1.05 & $1.8 \times 10^{5}$ & Bacillus sp. & 99 \\
J1-9 & MRS & 1.03 & $2.1 \times 10^{4}$ & Bacillus sp. & 99 \\
\hline
\end{tabular}

$\mathrm{mM}$ of SAM. In addition, a total of 11 isolated strains including salted toha shrimp (J1-9), salted herring roe (J43 ), salted scallop (J5-9), salted pen-shell (J7-10), salted hairtail (J8-3, J8-4), salted gizzard shad (J9-8), and salted branchia (J13-4) that produced about $1.00 \mathrm{mM}$ SAM were identified. Among 169 strains, 10 strains with excellent SAM production were selected from strains that had originated from each jeotgal. The results were similarly showed a previous study as SAM product strains from Kimchi. The strains isolated from the fermented kimchi products, produced the amount of SAM 1.22-1.58 mM (Lee et al, 2008). To utilize these strains in future studies, it was considered necessary to examine the characteristics and identification of these 10 strains.

\section{Sugar fermentation of strains}

For the 9 strains identified as Bacillus genus, an experiment was performed using an API 50 CHB system kit (Table 6) and for the J6-9 strain identified as Enterococcus genus, the sugar fermentation was measured using an API 20 Strep kit (Table 5). In the Delgado and Mayo's study, the differences Enterococcus sp. between strains were observed for glycerol, L-arabinose, D-xylose, mannitol, sorbitol, N-methyl-D-glucoside, amygdaline, mellibiose, sucrose, trehalose, melezitose, starch, $\beta$-gentibiose, tagatose, and gluconate (Delgado and Mayo, 2004). As a result, the Enterococcus durans genus strain showed sugar fermentation ability with ribose, lactose, trehalose, and raffinose, among 10 carbohydrates in the API 20 Strep kit. Each of the remaining 9 jeotgal-originated strains in the Bacillus genus had different substrates for fermentation depending on the strain, but they also showed strong fermentation in substrates such as glucose, fructose, mannose, glycerol, sucrose, trehalose, maltose, and N-acetylglucosamine. Although the difference among fermentable substrates was not great for each strain, it is considered that these strains seem to have enzymes to use many sug- ars and can ferment various sugars available in the body through food intake.

\section{Acid resistance $\&$ bile resistance}

Among the probiotic characteristics of the 10 strains isolated from jeotgal, acid resistance and bile resistance were examined. The gastric $\mathrm{pH}$ in healthy humans could be as low as about 2-2.5 (Fernandez et al., 2003) or high as pH 6 or above after food intake (Erikkila and Petaja, 2000). It was difficult to screen strains with strong acid resistance because strains could not grow below pH 5.0, but grew above $\mathrm{pH}$ 5.0. Most strains showed resistance to weak acids; particularly, J1-9 and J7-10 strains grew similarly at pH 5.0 compared to the control group at a neutral $\mathrm{pH}$, and the remaining strains could grow at $\mathrm{pH}$ 5.5. The strains showed weak resistance to strong acid but some resistance to a weaker acid of about pH 5.5 (Table 6). As for bile resistance, all 10 strains showed similar growth compared to the control group, suggesting that these strains have strong bile resistance. The structure of bacterial membrane can be disorganized by bile salt. So, bile salt tolerance is one of the essential properties for probiotic bacteria to survive (Lee and Salminen, 1995; Succi et al., 2005).

\section{Antibacterial activity}

The antibacterial activity was measured for five indicators such as Escherichia coli O157:H7, Enterococcus faecalis, Salmonella choleraesuis, Staphylococcus aureus, and Listeria monocytogenes using jeotgal-originated strains, which showed that the antibacterial activity was not observed by paper disc method. Instead, the antibacterial activity for the five indicators was partially identified in 21 strains with the exception of two strains, J7-1 and J8-3, through a soft agar method (Table 7). In particular, it is considered that J4-3 has strong antibacterial activity for En. faecalis because it had a relatively wide, 
Table 5. Sugar fermentation characteristics of strains with excellent SAM production (Bacillus spp.)

\begin{tabular}{|c|c|c|c|c|c|c|c|c|c|}
\hline Carbohydrate & J1-9 & J4-3 & J5-9 & J7-1 & J7-5 & J7-10 & J8-3 & J8-4 & J13-4 \\
\hline Control & $-^{*}$ & - & - & - & - & - & - & - & - \\
\hline Glycerol & ++ & - & + & ++ & ++ & ++ & + & + & + \\
\hline Erythritol & - & - & - & - & + & - & - & - & - \\
\hline D-arabinose & - & - & - & - & + & - & - & - & - \\
\hline L-arabinose & - & - & - & - & ++ & ++ & - & + & ++ \\
\hline Ribose & - & - & + & - & + & + & - & + & ++ \\
\hline D-xylose & - & - & - & - & - & - & - & - & - \\
\hline L-xylose & - & - & - & - & - & - & - & - & - \\
\hline Adonitol & - & - & - & - & - & - & - & - & - \\
\hline Methyl-B-xylopyranoside & - & - & - & - & - & + & - & - & - \\
\hline Galactose & - & - & + & - & + & + & + & + & ++ \\
\hline Glucose & ++ & - & ++ & ++ & ++ & ++ & ++ & ++ & ++ \\
\hline Fructose & ++ & - & ++ & ++ & ++ & ++ & ++ & ++ & ++ \\
\hline Mannose & ++ & - & ++ & ++ & ++ & ++ & ++ & ++ & ++ \\
\hline Sorbose & - & - & - & - & - & + & - & - & - \\
\hline Rhamnose & - & - & - & - & - & - & - & - & - \\
\hline Dulcitol & - & - & - & - & - & - & - & - & - \\
\hline Inocitol & + & & & + & ++ & - & + & - & - \\
\hline Mannotol & - & - & - & - & ++ & ++ & ++ & ++ & ++ \\
\hline Sorbitol & - & - & - & - & ++ & ++ & ++ & ++ & - \\
\hline Methyl- $\alpha$-D-mannopyranoside & - & - & - & - & - & - & - & - & ++ \\
\hline Methyl- $\alpha$-D-glucoside & - & - & - & - & + & ++ & + & + & + \\
\hline N-acetyl-glucosamine & + & - & + & + & + & ++ & + & + & ++ \\
\hline Amygdalin & + & - & + & + & - & ++ & + & + & + \\
\hline Arbutin & - & - & - & - & - & ++ & + & + & ++ \\
\hline Esculin & ++ & - & ++ & ++ & - & ++ & ++ & ++ & ++ \\
\hline Salicin & + & - & - & + & - & ++ & ++ & + & ++ \\
\hline Cellobiose & ++ & - & ++ & ++ & - & ++ & ++ & ++ & ++ \\
\hline Maltose & + & - & + & + & ++ & ++ & ++ & ++ & ++ \\
\hline Lactose & + & - & - & + & - & ++ & ++ & ++ & ++ \\
\hline Melibiose & - & - & + & - & + & - & + & + & ++ \\
\hline Sucrose & ++ & - & ++ & ++ & ++ & ++ & ++ & ++ & ++ \\
\hline Trehalose & + & & ++ & + & ++ & ++ & ++ & ++ & ++ \\
\hline Inulin & - & - & - & - & + & - & - & - & - \\
\hline Melezitose & - & - & - & - & - & - & - & - & - \\
\hline Raffinose & + & - & - & + & - & - & - & + & ++ \\
\hline Starch & - & - & - & - & - & - & - & ++ & - \\
\hline Glycogen & - & - & - & - & - & - & - & ++ & - \\
\hline Xylitol & - & - & + & - & - & - & - & - & - \\
\hline Gentiobiose & + & - & - & + & - & - & + & + & ++ \\
\hline D-turanose & - & - & - & - & + & - & - & - & - \\
\hline D-lyxose & - & - & - & - & - & - & - & - & - \\
\hline D-tagatose & - & - & - & - & - & + & - & - & - \\
\hline D-fucose & - & - & - & - & - & - & - & - & - \\
\hline L-fucose & - & - & - & - & - & - & - & - & - \\
\hline D-arabitol & - & - & - & - & - & - & - & - & - \\
\hline L-arabitol & - & - & - & - & - & - & - & - & - \\
\hline Gluconate & - & - & - & - & - & - & - & - & - \\
\hline 2-keto-gluconate & - & - & - & - & - & + & - & - & - \\
\hline 5-keto-gluconate & - & - & - & - & - & - & - & - & - \\
\hline
\end{tabular}

*++: strong, +: positive, - : negative

clear zone around the colony, and the J7-5 strain strongly inhibited Sal. choleraesuis. There was no strain that inhi- bited all five indicators, but two strains (J1-9, J7-10) inhibited at least four indicator strains, showing bacteria- 
Table 6. Acid resistance and bile resistance of SAM-producing strains

\begin{tabular}{cccccccc}
\hline \hline \multirow{2}{*}{ Strain } & & \multicolumn{3}{c}{ Acid resistance (pH) } & \multicolumn{3}{c}{ Bile resistance } \\
oxgall
\end{tabular}

*+: positive, -: negative

Table 7. Antibacterial activity of strains with excellent SAM production

\begin{tabular}{|c|c|c|c|c|c|}
\hline Strain & E. coli ${ }^{1)}$ & En. Faecalis ${ }^{2)}$ & (Sal. Choleraesuis ${ }^{3}$ & Sta. aureus $^{4)}$ & L. monocytogenes $^{3}$ \\
\hline J1-9 & $+^{*}$ & - & + & + & + \\
\hline $\mathrm{J} 4-3$ & + & ++ & - & - & - \\
\hline J5-9 & + & - & - & + & - \\
\hline J6-9 & - & - & - & + & + \\
\hline $\mathrm{J} 7-1$ & - & - & - & - & - \\
\hline $\mathrm{J} 7-5$ & - & - & ++ & + & + \\
\hline $\mathrm{J} 7-10$ & + & + & - & + & + \\
\hline $\mathrm{J} 8-3$ & - & - & - & - & - \\
\hline $\mathrm{J} 8-4$ & - & - & + & - & - \\
\hline $\mathrm{J} 13-4$ & + & + & - & - & - \\
\hline
\end{tabular}

*++: strong, +: positive, ${ }^{1)}$ E. coli; Escherichia coli $\mathrm{O} 157: \mathrm{H} 7,{ }^{2)}$ En. faecalis; Enterococcus faecalis, ${ }^{3)}$ Sal. choleraesuis; Salmonella choleraesuis, ${ }^{4}$ Sta. aureus; Staphylococcus aureus, ${ }^{5)}$ L. monocytogenes; Listeria monocytogenes

inhibiting ability for several indicators. J1-9 and J7-10 were identified Bacillus sp. and Bacillus amyloliquefaciens, respectively. Strains of Bacillus sp. have been studied as antibacterial activity of plant pathogens (Cook et al., 1995; Mari et al., 1996). Among the strains with acid resistance mentioned above, J1-9 and J7-10 could grow at the lowest $\mathrm{pH}$ and showed a strong inhibition to indicators, suggesting that these may be considered the most beneficial strains with a strong efficacy of probiotics.

These strains are considered as strains that can be involved in the regulation of the intestines. In terms of fermentation characteristics, the results of the substrate utilization of seven strains showed that fermentable substrates vary depending on the strains and thus, strains seem to have enzymes for using sugars and can ferment various sugars in the body through food intake. Finally, strains showed weak acid resistance but excellent bile resistance and antibacterial activity; thus, it is considered that in the future, various strains originating from jeotgal can be used as starters in fermented foods to provide SAM and probiotics in food. Also, it is expected that these strains can be used in the development of after-meal desserts, considering the characteristics of the stomach, which contains higher $\mathrm{pH}$ levels with a full stomach compared to an empty stomach, due to acid resistance to weak acids, and can be utilized in foods using strain coating such as capsules to supplement acid resistance.

\section{Conclusion}

This study was performed to isolate fermented salted seafood-originated strains select and identify strains that produce larger amounts of SAM in food. The production of SAM was measured in 169 strains from 18 kinds of jeotgal and, after the identification of these strains, most belonged to the Bacillus genus. Particularly among strains isolated from salted pen-shell, 3 strains showed over 1.0 $\mathrm{mM}$ of SAM production. Among probiotic characteristics, acid resistance and bile resistance were examined in 10 strains with more than $1.0 \mathrm{mM}$ of SAM production; most strains could grow in the weak acidic condition over $\mathrm{pH}$ 5.5, and only $\mathrm{J} 1-9$ and $\mathrm{J} 7-10$ strains could grow below $\mathrm{pH}$ 5.0 , while no resistance was observed in any of the strains below $\mathrm{pH}$ 4.5. On the other hand, for bile resistance, all 
strains showed similar growth compared to the control group, suggesting that these strains have bile resistance and, thus, probiotics characteristics. The results of antibacterial activity for five indicators such as Escherichia coli O157:H7, Enterococcus faecalis, Salmonella choleraesuis, Staphylococcus aureus, and Listeria monocytogenes in the strains originating from the jeotgal showed that the antibacterial activity was not observed in the paper disc method and that 2 out of 10 strains did not show the inhibitory activity in the soft agar method, but the remaining 8 strains showed strong or excellent antibacterial effects.

\section{Acknowledgements}

This study was supported by a grant from the Korea Food Research Institute (Project No. E0131301).

\section{References}

1. Burren, K. A., Mills, K., Copp, A. J., and Greene, N. D. (2006) Quantitative analysis of S-adenosylmethionine and S-adenosyl-homocysteine in neurulation-stage mouse embryos by liquid chromatography tandem mass spectrometry. J. Chromatogr. B. 844, 112-118.

2. Cook, R. J., Thomashow, L. S., Weller, D. M., Fujimoto, D., Mazzola, M., Bangera, G., and Kim, D. S. (1995) Molecular mechanisms of defense by rhizobacteria against root disease. P. Natl. Acad. Sci. USA. 92, 4197-4201.

3. Cooney, C. A. (1993) Are somatic cells inherently deficient in methylation metabolism? A proposed mechanism for DNA methylation loss, senescence and aging. Growth Dev. Aging 57, 261-273.

4. Corrales, F., Giménez, A., Alvarez, L., Caballería, J., Pajares, M. A., Andreu, H., Parés, A., Mato, J. M., and Rodés, J. (1992) $\mathrm{S}$-adenosylmethionine treatment prevents carbon tetrachloride-induced S-adenosylmethionine synthetase inactivation and attenuates liver injury. Hepatology 16, 1022-1027.

5. Delgado, S. and Mayo, B. (2004) Phenotypic and genetic diversity of Lactococcus lactis and Enterococcus spp. Strains isolated from Northern Spain starter-free farmhouse cheeses. Int. J. Food Microbiol. 90, 309-319.

6. Erkkila, S. and Petaja, E. (2000) Screening of commercial meat starter cultures at low $\mathrm{pH}$ and in the presence of bile salts for potential probiotic use. Meat Sci. 55, 279-300.

7. Fernandez, M. F., Boris, S., and Barbes, C. (2003) Probiotic properties of human lactobacilli strains to be used in the gastrointestinal tract. J. Appl. Microbiol. 94, 449-455.

8. Guattari B. (1991) High-performance liquid chromatographic determination, with ultraviolet detection, of S-adenosyl-Lmethionine and of normetanephrine and metanephrine for phenylethanolamine-N-methyltransferase or catechol-O-methyltransferase activity. J. Chromatogr. 567, 254-260.
9. Horikawa, S., Sasuga, J., Shimizu, K., Ozasa, O., and Tsukada, K. (1990) Molecular cloning and nucleotide sequence of cDNA encoding the rat kidney S-adenosylmethionine synthetase. $J$. Biol. Chem. 23, 13683-13686.

10. Hur, S. H. (1996) Critical review on the microbiological standardization of salt-fermented fish product. J. Korean Soc. Food Sci. Nutr. 25, 885-891.

11. Kim, J. Y., Seo, H. S., Seo, M. J., Suh, J. W., Hwang, I., and Ji G. E. (2008) Development of S-adenosyl-L-methionine (SAM)-reinforced probiotic yogurt using Bifidobacterium bifidum BGN4. Food Sci. Biotechnol. 5, 1025-1031.

12. Koning, B. (1987) A long-term (two years) clinical trial with $\mathrm{S}$-adenosyllmethionine for the treatment of osteoarthritis. Am. J. Med. 20, 89-94.

13. Lee, K. H. (1969) Microbiological and enzymological studies on the flavor components of sea food pickles. J. Korean Agric. Chem. Soc. 11, 1-6.

14. Lee, M. K., Lee, J. K., Son, J. A., Kang, M. H., Koo, K. H., and Suh, J. W. (2008) S-adenosyl-L-methionine (SAM) production by lactic acid bacteria strains isolated from different fermented kimchi products. Food. Sci. Biotechnol. 17, 857860.

15. Lee, N. K., Jeon, E. H., Lee, H. J., Cho, I. J., and Hahm, Y. T. (2006) Isolation, identification, and characterization of Bacillus spp. from the traditionally fermented cheonggukjangs in the Gyeonggi and the Gangwon provinces. J. Korean. Soc. Appl. Biol. Chem. 49, 276-280.

16. Lee, Y. K. and Salminen, S. (1995) The coming of age of probiotics. Trends Food Sci. Technol. 6, 241- 245.

17. Lieber, C. S. (1999) Role of S-adenosyl-L-methionine in the of treatment of liver diseases. J. Hepatol. 30, 1155-1159.

18. Mari, M., Guizzardi, M., Brunelli, M., and Folchi, A. (1996) Postharvest biological control of grey mould (Bortytis cinerea Pers.: Fr.) on fresh-market tomatoes with Bacillus amyloliquefaciens. Crop Prot. 15, 699-705.

19. Mato, J. M., Camara, J., Fernandez de Paz, J., Caballeria, L., Coll, S., Caballero, A., Garcia-Buey, L., Beltran, J., Benita, V., Caballeria, J., Solas, R., Moreno-Otero, R., Barrao, F., Martin-Duce, A., Correa, J. A., Pares, A., Barrao, E., GarciaMagaz, I., Puerta, J. L., Moreno, J., Boissardg, G., Ortiz, P., and Rodes, J. (1999) S-Adenosylmethionine in alcoholic liver cirrhosis: a randomized, placebo-controlled, double-blind, multicenter clinical trial. J. Hepatol. 30, 1081-1089.

20. Porter, C. W. and Sufrin, J. R. (1986) Interference with polyamine biosynthesis and/or function by analogs of polyamines or methionine as a potential anticancer chemotherapeutic strategy. Anticancer Res. 6, 525-542.

21. Sands, A. and Crisan, E. V. (1974) Microflora of fermented Korean seafoods. J. Food Sci. 39, 1002-1005.

22. Santi, R. D. and Rodegerdts, U. (1983) Functional myelography in spondylolisthesis. Arch. Ortho. Trauma. Surg. 101, 7582.

23. Shelly, C. L. (2000) S-adenosylmethionine. Int. J. Biochem. Cell Bio. 32, 391-395.

24. Succi, M., Tremonte, P., Reals, A., Sorrentino, E., Grazia, L., and Pacifico, S. (2005) Bile salt and acid tolerance of Lacto- 
bacillus rhamnosus strains isolated from Parmigiano Reggiano cheese. FEMS Microbiol. Lett. 244, 129-137.

25. Wang, W., Kramer, P. M., Yang, S., Pereira, M. A., and Tao, L. H. (2001) Reversed-phase high-performance liquid chromatography procedure for the simultaneous determination of
S-adenosyl-L-methionine and S-adenosyl-L-homocysteine in mouse liver and the effect of methionine on their concentrations. J. Chromatogr. 762, 59-65.

(Received 2013.8.19/Revised 2014.1.5/Accepted 2014.1.20) 